\section{Cancer Treatment}

Single Agents in Cancer Chemotherapy. By Robert B. Livingston and Stephen K. Carter. Pp. $x+405$. (IFI/Paleum: New York and London, 1970.) \$20.

THIs book brings together and summarizes the results of clinical trials conducted since 1955 by the National Cancer Institute of the United States on the effects of certain anti-cancer agents given to patients with a range of specified types of malignant disease. As the title implies, the data all concern the effects of the agents given alone rather than in combination with other drugs or with other forms of therapy. The information is presented in tabular form and is arranged for each drug according to type of disease, and numbers and percentages of patients responding to stated dose schedules. Sixteen drugs have entire chapters devoted to them. These are nitrogen mustard (HN2), cyclophosphamide, chlorambucil, melphalan, busulfan, methotrexate, 6-mercaptopurine, 5fluorouracil, cytosine arabinoside, hydroxyurea, actinomycin $\mathrm{D}$, mithramycin, vinblastine, vincristine, procarbazine and prednisone (and prednisolone). Data from trials on another nine agents are considered more briefly.

Only highly selected data have been summarized. For inclusion, reports had to fulfil certain medical and scientific criteria and, with few exceptions, to be published in the English language.

The principal value of the book lies in the brief comments on the role in cancer therapy of each of the agents reviewed. Experienced chemotherapists may not agree with some of the conclusions-for example, the overall response rate (22 per cent) of patients with melanoma to dimethyl triazeno imidazole carboxamide compares favourably with that to any other agent used against this form of malignant disease. Nevertheless, such statements provide a most useful background against which to judge the effects of new agents and new regimens.

The book holds little to interest those not working directly in the field of cancer chemotherapy, although reading it might engender in the mind of the non-specialist the thought that, if there existed for the more common types of cancer more effective anti-cancer agents, the need for painstaking comparisons of only weakly effective treatments might largely disappear.

Francis J. C. RoE

\section{Lipid Handbook}

Lipid Metabolism. Edited by S. J. Wakil. Pp. xi+613. (Academic: New York and London, January 1971.) $\$ 28.50 ; £ 13.30$.

THIs book contains a collection of contributions on major topics in the field of lipid biochemistry by contributors who, with the sad exception of the late Professor G. Hübscher, are all actively engaged in the forefront of their respective fields of research. The editor aims to provide a comprehensive account of major achievements and trends in the field for investigators, teachers and students. The need for this undoubtedly exists, for it is more than a decade since the last comprehensive reference book on lipid metabolism was published (Lipide Metabolism, edited by K. Bloch, J. Wiley and Sons, New York, 1960). In an area of biochemistry where there has been formidable growth, ten years is far too long.

The titles of the chapters suggest a textbook thoroughness in their selection, which is not altogether typical of their contents. As one would expect, there are chapters on the metabolism of fatty acids, sterols, glycerides and phospholipids, as well as more specialized contributions on bacterial lipids, on prostaglandin metabolism and on the biosynthesis of aromatic amines and of polyisoprenoid quinones. The contributions themselves suggest that the writers had considerable freedom in their choice of presentation. Several contributors have chosen to discuss their own interests at length, treating other topics much more concisely. Thus, in his chapter on fatty acid metabolism, Wakil gives an extensive account of the enzymology of fatty acid synthesis and the numerous possibilities of its control, but only briefly describes the enzymology of fatty acid oxidation. Inevitably, this kind of presentation is rather unbalanced, but to my mind the disadvantages are usually redressed by the interest of the more detailed sections. Textbooks are rarely stimulating. Other contributors have used a more balanced approach. Hübscher, for instance, has written an exemplary review on glyceride metabolism which will undoubtedly be of great use to students, teachers and research workers.

In a single volume covering such a large field there will inevitably be some topics which are left out. In the chapters dealing with the metabolism of lipids with cyclic structures, and in the chapter on prostaglandins, the emphasis is strongly on the mechanisms of reactions: it is necessary to look elsewhere for information about regulatory phenomena and about the physiological function of some of the compounds discussed. It seems a pity that Samuelsson decided to omit even the briefest discussion on the biological functions of prostaglandins. In this respect, fatty acid metabolism is better served by Wakil and Bressler and glyceride metabolism by Hübscher. All three contributors provide a broader biological perspective for their subjects. I felt that another omission was a chapter on the role of lipids in membranes, in view of the current interest in this topic.
Lennarz makes some interesting comments on the subject, principally with regard to bacterial membranes, but that is all. Doubtless Wakil will remedy this omission if his promised second volume comes into being.

In summary, Lipid Metaholism, covering the literature to the end of 1969 , is a much-needed book. That it does not cover all aspects of lipid biochemistry is not surprising in view of its size. In any case the amount of information present makes the book an essential acquisition for anyone interested in the field. Much of the contents is stimulating and challenging. I found the phospholipid chapter particularly so. Towards the end of the book the reader may find himself hastening to look up the definition of a lipid. He may then wonder at the elegant and precise approaches that have been used to study such an ill-defined group of compounds. JILL ILIFFE

\section{Immune Machinery}

Immune Surveillance: Perspectives in Immunology. Edited by Richard T. Smith and Maurice Landy. Pp. xvi +536 . (Academic: New York and London, March 1971.) $£ 5.60$.

IN each of the past three years, a conference, under the sponsorship of the National Institute of Allergy and Infectious Diseases, has been held at Brook Lodge, Augusta, Michigan. It is in fact a sort of immunological masters' tournament--the entry is restricted to those who have done well in previous years and the few promising youngsters whose performance on the circuit augurs well for their future. A few distinguished but less active seniors are asked along for the occasional comment. All that is lacking is the excited audience and the hot-dog sellers.

The book which is the subject of this review provides the link between the conference and a wider public, but unfortunately it lacks the dramatic impact of the occasion itself; to continue the golfing analogy, it is akin to an edited television recording. I would also dispute the claim that the conference was in any sense an informal occasion. The very fact that a record was made -as far as can be judged very efficiently by stenotypy - in the presence of a distinguished group of scientists effectively precludes informality. Nevertheless there must be no doubt that this book records an important occasion in the world of immunology.

The most significant item is the statement and discussion of Jerne's new theory on the generation of antibody diversity and self-tolerance. The editors have skilfully summarized the notions presented in the form of a flow sheet, and indeed throughout the book their inter- 